\title{
Periscopic technique in Norwood operation is associated with better preservation of early ventricular function
}

Puneet Bhatla, MD, ${ }^{\mathrm{a}, \mathrm{b}}$ TK Susheel Kumar, MD,${ }^{\mathrm{c}}$ Luv Makadia, MD,${ }^{\mathrm{d}}$ Brandon Winston, MPH, ${ }^{\mathrm{c}}$ Catherine Bull, PNP-BC, ${ }^{\mathrm{c}}$ James C. Nielsen, MD, ${ }^{\mathrm{a}}$ David Williams, MD, ${ }^{\mathrm{c}}$ Sujata Chakravarti, MD, ${ }^{\mathrm{a}}$ Richard G. Ohye, MD, ${ }^{e}$ and Ralph S. Mosca, $\mathrm{MD}^{\mathrm{c}}$

\section{ABSTRACT}

Objective: Although the right ventricle (RV) to pulmonary artery conduit in stage 1 Norwood operation results in improved interstage survival, the long-term effects of the ventriculotomy used in the traditional technique remain a concern. The periscopic technique (PT) of RV to pulmonary artery conduit placement has been described as an alternative technique to minimize RV injury. A retrospective study was performed to compare the effects of traditional technique and PT on ventricular function following Norwood operation.

Methods: A retrospective study of all patients who underwent Norwood operation from 2012 to 2019 was performed. Patients with baseline RV dysfunction and significant tricuspid valve regurgitation were excluded. Prestage 2 echocardiograms were reviewed by a blinded experienced imager for quantification of RV function (sinus and infundibular RV fractional area change) as well as for regional conduit site wall dysfunction (normal or abnormal, including hypokinesia, akinesia, or dyskinesia). Wilcoxon rank-sum tests were used to assess differences in RV infundibular and $\mathrm{RV}$ sinus ejection fraction and the Fisher exact test was used to assess differences in regional wall dysfunction.

Results: Twenty-two patients met inclusion criteria. Eight underwent traditional technique and 14 underwent PT. Median infundibular RV fractional area change was $49 \%$ and $37 \%(P=.02)$ and sinus RV fractional area change was $50 \%$ and $41 \%$ for PT and traditional technique $(P=.007)$ respectively. Similarly qualitative regional $\mathrm{RV}$ wall function was better preserved in $\mathrm{PT}(P=.002)$.

Conclusions: The PT for RV to pulmonary artery conduit in Norwood operation results in better preservation of early RV global and regional systolic function. Whether or not this benefit translates to improved clinical outcome still needs to be studied. (JTCVS Techniques 2021;8:116-23)

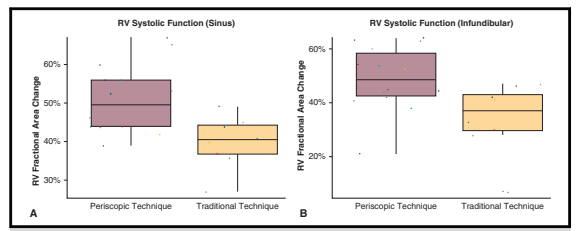

Boxplot comparison of periscopic versus traditional technique for RV systolic function.

\section{CENTRAL MESSAGE}

Use of periscopic technique for right ventricle to pulmonary conduit placement in stage 1 Norwood operation is associated with better preservation of right ventricular function.

\section{PERSPECTIVE}

Although right ventricle to pulmonary artery conduit in Norwood operation results in improved interstage survival, the long-term adverse effects of ventriculotomy remain a concern. The periscopic technique in which a graft is placed inside from within the ventricle combines the advantages of right ventricle to pulmonary artery conduit along with preservation of ventricular function

See Commentary on page 124 .
From the ${ }^{\mathrm{a}}$ Division of Pediatric Cardiology and Departments of ${ }^{\mathrm{b}}$ Radiology, ${ }^{\mathrm{c}} \mathrm{Cardiac}$ Surgery, and ${ }^{\mathrm{d}}$ Pediatrics, New York University Langone Medical Center, New York, NY; and 'Department of Cardiac Surgery, Mott Children's Hospital, Ann Arbor, Mich.

Accepted for the 46th Annual Meeting of the Western Thoracic Surgical Association, Vail, Colorado, June 24-27, 2020.

Received for publication July 31, 2020; accepted for publication May 13, 2021; available ahead of print May 21, 2021.

Address for reprints: Ralph S. Mosca, MD, Department of Cardiac Surgery, NYU Langone Medical Center, 530 First Ave, Suite 9v, New York, NY 10016 (E-mail: ralph.mosca@nyulangone.org).

2666-2507

Copyright $@ 2021$ The Authors. Published by Elsevier Inc. on behalf of The American Association for Thoracic Surgery. This is an open access article under the CC BY-NC-

ND license (http://creativecommons.org/licenses/by-nc-nd/4.0/).

https://doi.org/10.1016/j.xjtc.2021.05.014 $\checkmark$ Video clip is available online.

Neonatal management for hypoplastic left heart syndrome (HLHS) has significantly evolved over the years. As part of multistaged palliation, the Norwood procedure still continues to be the most commonly performed and favored option for stage 1 palliation. The goals of the procedure are to provide unobstructed systemic blood flow from the single 


$$
\begin{aligned}
& \text { Abbreviations and Acronyms } \\
& \text { EDA }=\text { end diastolic area } \\
& \text { FAC }=\text { fraction area change } \\
& \text { HLHS = hypoplastic left heart syndrome } \\
& \text { MBTT = modified Blalock-Taussig-Thomas } \\
& \text { PT = periscope technique } \\
& \mathrm{RV} \quad=\text { right ventricle } \\
& \mathrm{RV}-\mathrm{PA}-\mathrm{C}=\text { right ventricle to pulmonary artery } \\
& \text { conduit } \\
& \text { TT }=\text { traditional technique }
\end{aligned}
$$

right ventricle (RV) and to provide a controlled source of pulmonary blood flow. Two approaches have been utilized to provide a reliable source of pulmonary blood flow; placement of a modified Blalock-Taussig-Thomas (MBTT) shunt or a RV to pulmonary artery conduit (RV-PA-C). Improved interstage survival and outcomes have been described in patients receiving RV-PA-C, when compared with those with MBTT shunt, making it a preferred choice for many surgeons. ${ }^{1-4}$ However, the RV incision, intrinsic to RV-PA-C procedure, has long-term consequences, including arrhythmias, pseudoaneurysm formation, and single ventricular dysfunction. ${ }^{5-8}$ The dunk technique, in which a ringed polytetrafluoroethylene conduit is inserted into the RV cavity from outside, has been described to offset some of these complications. ${ }^{9}$ However, this can be technically challenging because the rings can often get caught onto the RV myocardium and result in injury when the graft is pushed into the RV. We described the periscopic technique (PT) of RV-PA-C placement, in which the graft is drawn from within the RV as an alternative to minimize the risk of possible ventricular tearing. ${ }^{10}$ Whether or not this advantage translates to preservation of ventricular systolic function is unknown. Therefore, we conducted a retrospective, single-center study to analyze the ventricular function in patients with HLHS who underwent stage 1 palliation using the PT and compared it with the traditional technique (TT) of RV-PA-C insertion.

\section{METHODS \\ Patients}

The study protocol was approved by the Institutional Review Board of New York University Langone Health (s 19-01559; November 8, 2019). This was a retrospective study and hence our institutional review board waived patient consent. The institutional cardiac database was used to retrospectively identify all patients who underwent Norwood operation between January 1, 2012, and April 1, 2019. All patients underwent operation performed by a single surgeon. Patients with prematurity, presence of extracardiac abnormalities, pre-Norwood RV systolic dysfunction, significant tricuspid valve regurgitation, and conduction abnormalities were excluded. Patients who did not have adequate echocardiographic images to assess the RV systolic function were also excluded from the study. A total of 36 patients were identified, from which 22 met inclusion criteria.

\section{Surgical Technique of RV-PA-C}

TT. In the early part of our practice, we used a $5 \mathrm{~mm}$ nonringed polytetrafluoroethylene graft (Gore-Tex; W.L. Gore and Associates, Flagstaff, Ariz) as RV-PA-C during the Norwood operation. An incision was made over the infundibulum of the RV and a small amount of muscle was resected. The beveled end of the graft was then sutured to this ventriculotomy using 5.0 Gore-Tex suture in a continuous manner. The distal end of the graft was then sutured to a fenestration within a patch of pulmonary homograft used to cover the distal end of the pulmonary bifurcation using continuous 5.0 Gore-Tex suture.

PT. The periscope modification of RV-PA-C placement in the Norwood operation has been well described by our group (Video 1$).{ }^{10}$ Following median sternotomy and creation of pericardial well, the distance between the planned site of ventriculotomy and the pulmonary artery bifurcation is measured. A 5-mm ringed Gore-Tex graft is trimmed to length. The proximal end of the graft is cut flush with a ring and the graft is marked usually at the third ring from the divided end to correspond to the thickness of the infundibular free wall. Cardiopulmonary bypass is instituted through arterial cannulation of the distal main PA and venous cannulation of the right atrium. The branch PAs are snared and systemic cooling to deep hypothermia is initiated. The ascending aorta, the head and neck branches, the isthmus, and the descending thoracic aorta are all well mobilized with due attention to preservation of the recurrent laryngeal nerve. The arterial cannula is then snared at the ductus arteriosus and the main PA is transected just proximal to the bifurcation. The PA bifurcation is patched with a thick portion of the pulmonary homograft tissue. An incision is then made in the infundibulum at the planned site using a No. 11 blade and gently stretched with a clamp. No RV muscle is resected. A right angle instrument is then passed through the incision, the RV outflow tract and out through the divided PA (see Figure 1). To safeguard the neoaortic valve, certain measures are adopted. The section of the graft with no rings is introduced first under direct vision to facilitate the initial retrieval of the graft. Once grasped, the reminder of the graft is placed within the RV under vision to avoid damage to the leaflets and gradually pulled in a retrograde manner. Using a portion of the graft without rings also facilitates its passage through the RV incision. The appropriately tailored and marked conduit is then pulled gently in a retrograde fashion through the RV stab wound until the mark is visible and the proximal end is flush with the endocardium. This can also be confirmed from within the RV by looking through the proximal main PA. The graft is then secured with an epicardial circumferential 6-0 polypropylene purse string. Following neoaortic arch reconstruction and atrial septectomy, the distal connection of the RV-PA-C is established by dunking it into a fenestration in the patch over the distal

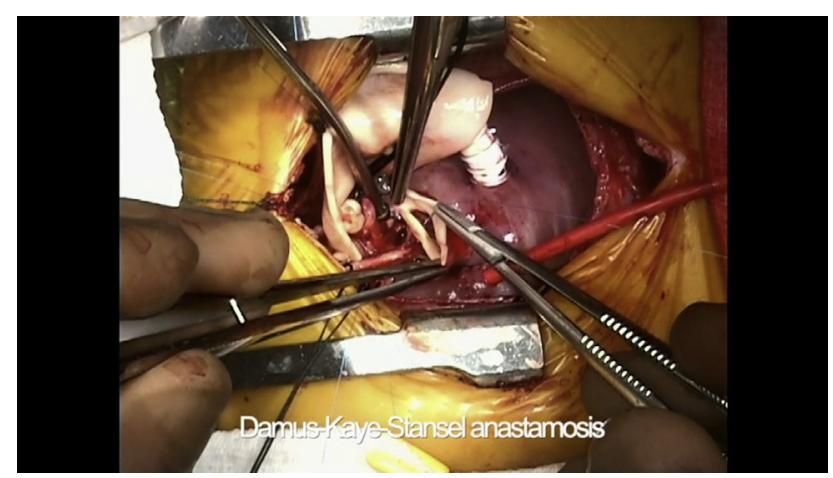

VIDEO 1. Video demonstrating periscopic technique of right ventricle to pulmonary artery conduit (RV-PA-C) placement in Norwood operation. Video available at: https://www.jtcvs.org/article/S2666-2507(21)00380-1/ fulltext. 


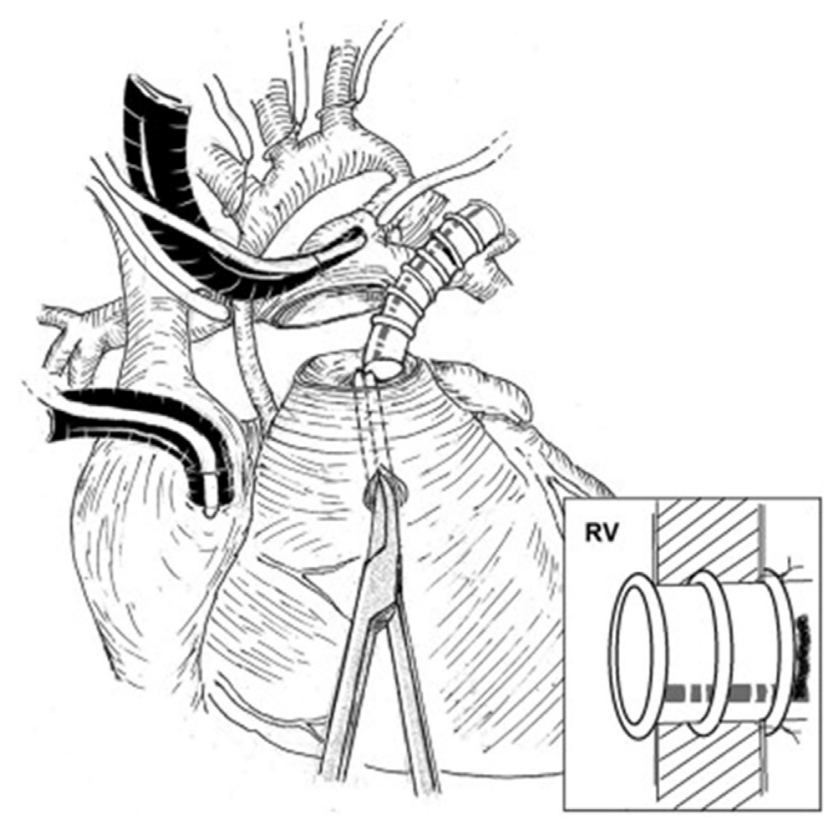

FIGURE 1. The graft is passed through the divided pulmonary artery out the right ventricle $(R V)$ with a right-angle clamp. Inset, A ring at the end of the graft anchors at the RV endocardium functioning as a hemostatic O-ring. The graft is secured with circumferential 6-0 polypropylene sutures onto the epicardium.

PA bifurcation. The distal connection is also secured by using a circumferential 6-0 polypropylene purse string. ${ }^{11}$

\section{Echocardiography}

All echocardiographic studies were performed by a single laboratory, using either an IE33 or Affinity (Phillips Medical Systems, Andover, Mass) echocardiographic system equipped with multiple frequency transducers. All data were digitally stored and measurements were performed offline using syngo Dynamics software (Siemens Healthineers, Munich, Germany). Prestage 2 echocardiograms were identified on patients who met inclusion criteria. Patients who had adequate echocardiographic images for assessment of single RV systolic function at the sinus level and also the infundibular region were included in the study. Specifically, patients who had echocardiographic images in an apical 4-chamber at the RV sinus level (Figure 2, A), RV infundibular level (coronal plane obtained by sweeping anteriorly from a standard 4 chamber plane) (Figure 2, B), and parasternal long (Figure 2, $C$ ) and short (Figure 2, D) axis images at the RV infundibular level were included in the study. Image quality was assessed to ensure adequate visualization of the blood-endocardial border. All image interpretation, measurements, and analysis were performed on retrospective imaging data by an experienced echocardiographer blinded to the clinical data. The parasternal images were used for evaluation of regional wall function at the RV-PA-C insertion site. Regional function was categorized as being normal (grade I) or abnormal (ie, mild to moderate hypokinesia [grade II], severe hypokinesia/akinesia [grade III], or dyskinesia [grade $\mathrm{VI}])$. The apical imaging planes were used to quantify RV function using the fractional area change (FAC) method at the sinus and infundibular levels. ${ }^{10} \mathrm{RV}$ end-diastolic area (RVEDA) and RV end-systolic area were measured at the RV sinus and RV infundibular levels. RV end-diastole and end-systole were not defined by electrocardiographic criteria; instead, they were defined by the time point at which the RV was the largest and smallest, respectively. All measurements for planimetered areas consistently included RV trabeculations and papillary muscles to minimize under- or overestimation of RVFAC. RVFAC was calculated for each patient, both at the sinus level and infundibular level using the following formula: (RVEDA $\left[\mathrm{cm}^{2}\right]-\mathrm{RV}$ end-systolic area $\left[\mathrm{cm}^{2}\right]$ )/RVEDA $\left(\mathrm{cm}^{2}\right)$ (Figure 3, $A$ and $B$ ).

\section{Statistical Analysis}

Continuous variables are presented as median (range). Baseline variables were compared between the TT and PT cohorts with the use of either a Wilcoxon rank-sum exact test (for continuous variables) or a Fisher exact test (for categorical variables). All statistical analyses were performed with the use of $\mathrm{R}$ software version 3.5.3 (R Foundation for Statistical Computing, Vienna, Austria).

\section{RESULTS \\ Participants}

A total of 38 patients were identified. Of these, 22 patients met inclusion criteria. See Table 1 for patient characteristics. Because the sample size is small, nonparametric methods were employed in the analysis of results. Out of the 22 patients, 8 underwent RV-PA-C insertion using the TT, whereas the remaining 14 patients underwent RV-PA$\mathrm{C}$ insertion using the PT. The median age at stage 1 palliation was 3.5 days (range, 2-18 days) for the TT group and 5.0 days (range, 3-18 days) for the PT group. PreNorwood procedure, all patients in both groups displayed normal RV function. Additionally, none of the patients in either group had significant tricuspid valve regurgitation. The median age for patients at the time of prestage 2 echocardiogram used for RV function assessment was 31.5 days (range, 13-134 days) in the TT group and 26.5 days (range, 13-261 days) for the PT group. Post-Norwood procedure, none of the patients in either group had any significant neoaortic valve regurgitation. The PT group experienced more postoperative arrhythmias, but this was not statistically significant $(P=.3512)$. There was no statistically significant difference in the number of catheter interventions between the 2 groups.

\section{RV Function}

The cohort of patients who underwent the PT showed a significant improvement over the cohort that underwent the TT. For the PT cohort, the median sinus RVFAC was $50 \%$ (range, 39\%-67\%) compared with $41 \%$ (range, $27 \%-49 \%)$ for the TT group $(P=.007)$ (Figure 4, $A$ ). The median infundibular RVFAC was also significantly better in the PT group, $49 \%$ (range, $21 \%-64 \%$ ) compared with the TT group, 37\% (range, $7 \%-47 \%)(P=.02)$ (Figure 4, B). Similarly, qualitative regional RV wall function was significantly better preserved in the PT group when compared with the TT group. Six out of the 8 patients to undergo the TT had regional RV wall dysfunction of grade 2 or greater, whereas only 1 out of the 14 patients to undergo the PT demonstrated grade 2 dysfunction $(P=.002)$. 

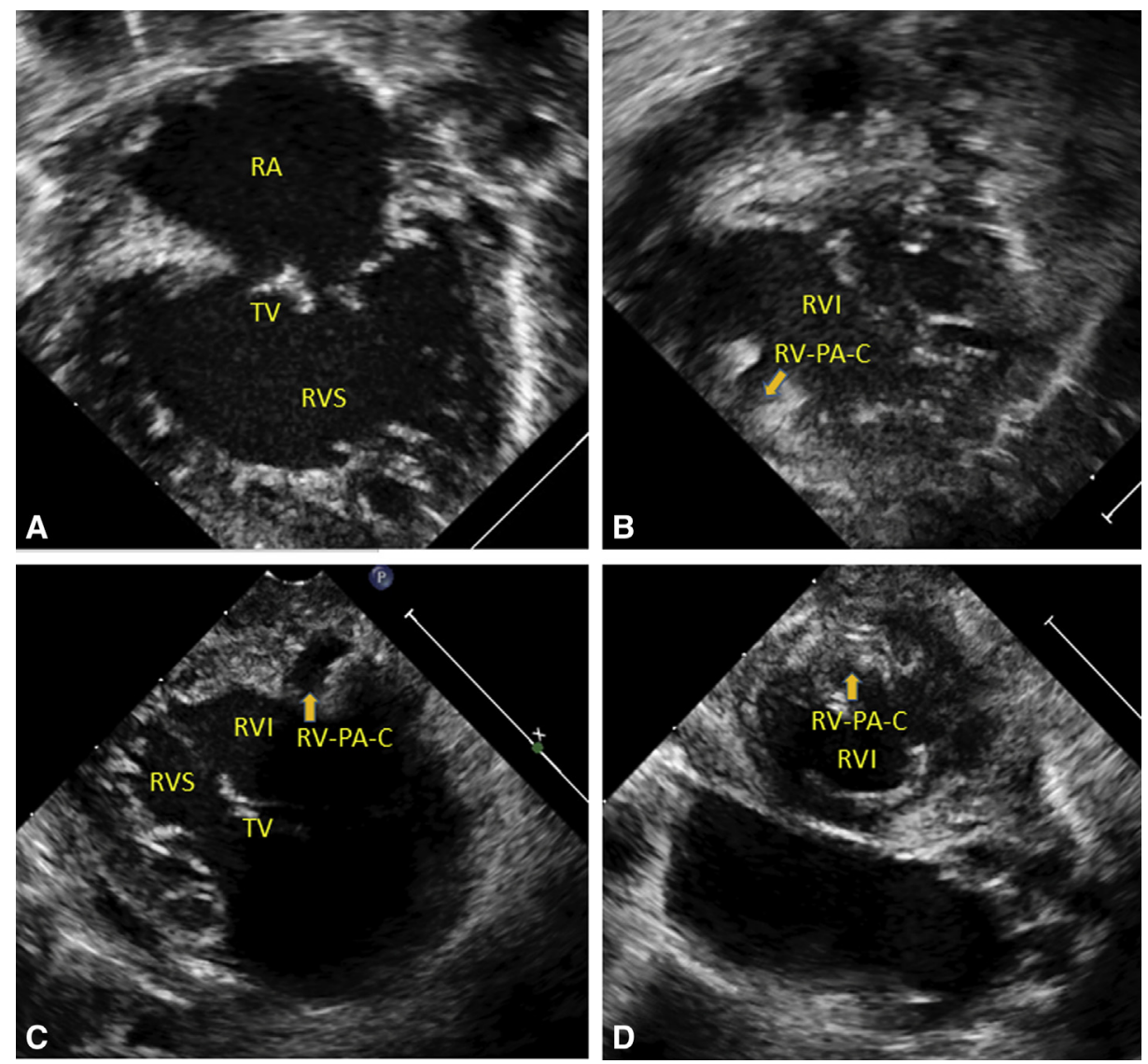

FIGURE 2. Right ventricle (RV) global and regional systolic function assessment. Apical 4-chamber image at the RV sinus (RVS) level (A) and RV infundibulum $(R V I)$ level (B), used for calculation of RV fractional area change (FAC). Parasternal imaging of the RV, at the RV to pulmonary artery conduit $(R V-P A-C)$ insertion site, both in long axis (C) and short axis (D), used for assessment of RV regional function. RA, Right atrium; $T V$, tricuspid valve.

\section{DISCUSSION}

The stage 1 Norwood palliation for HLHS has undergone numerous modifications, particularly in the technique of RV-PA-C placement. The PT, as a modification of the dunk technique, was described by our group to minimize ventricular injury and the resultant deleterious effects on ventricular systolic function. This study confirms our hypothesis that the PT results in improved preservation of RV systolic function.

In 2003, Sano and colleagues ${ }^{4}$ reported improved outcomes with the use of RV-PA-C as a source of pulmonary blood flow during Norwood stage 1 operation. The Single Ventricle Reconstruction trial confirmed an improvement in transplantation-free survival at 12 months amongst the group that received the RV-PA-C, compared with the MBTT shunt group. ${ }^{1}$ However, a follow-up of the study patients revealed that by 3 years, the transplantation-free survival was no longer significantly different, a change that has been attributed to either the incision over the RV or volume overload from the free insufficiency of a valveless conduit. ${ }^{12,13}$ Various modifications and innovative techniques have been described to potentially overcome these shortcomings. Kumar and colleagues ${ }^{14}$ described the use of valved RV-PA-C in the form of femoral vein homograft to minimize the effects of free insufficiency over RV function; however, the femoral veins are associated with risk of significant reintervention during the interstage period. In an attempt to minimize the effects of ventricular incision on $\mathrm{RV}$ function, Tweddell and colleagues ${ }^{9}$ described the dunk technique without removal of any muscle for proximal construction of RV-PA-C. Although this technique limits the ventriculotomy, its outside-in approach can be technically challenging and the rings often can get caught on the RV myocardium resulting in RV injury. Moreover, perhaps related to these inherent difficulties of this technique, the use of this modification did not translate into improved RV function. ${ }^{15}$ In our modification of RV-PA shunt, which we call PT, the ringed graft is inserted through the PA into the RV and then subsequently out of the RV in a prograde fashion, which allows for a limited ventriculotomy and eliminating the need for myectomy, and hence minimizing the risk of myocardial injury. ${ }^{10}$ Moreover, only epicardial securing sutures are necessary for our technique as opposed to the TT where broader and deeper suturing is 


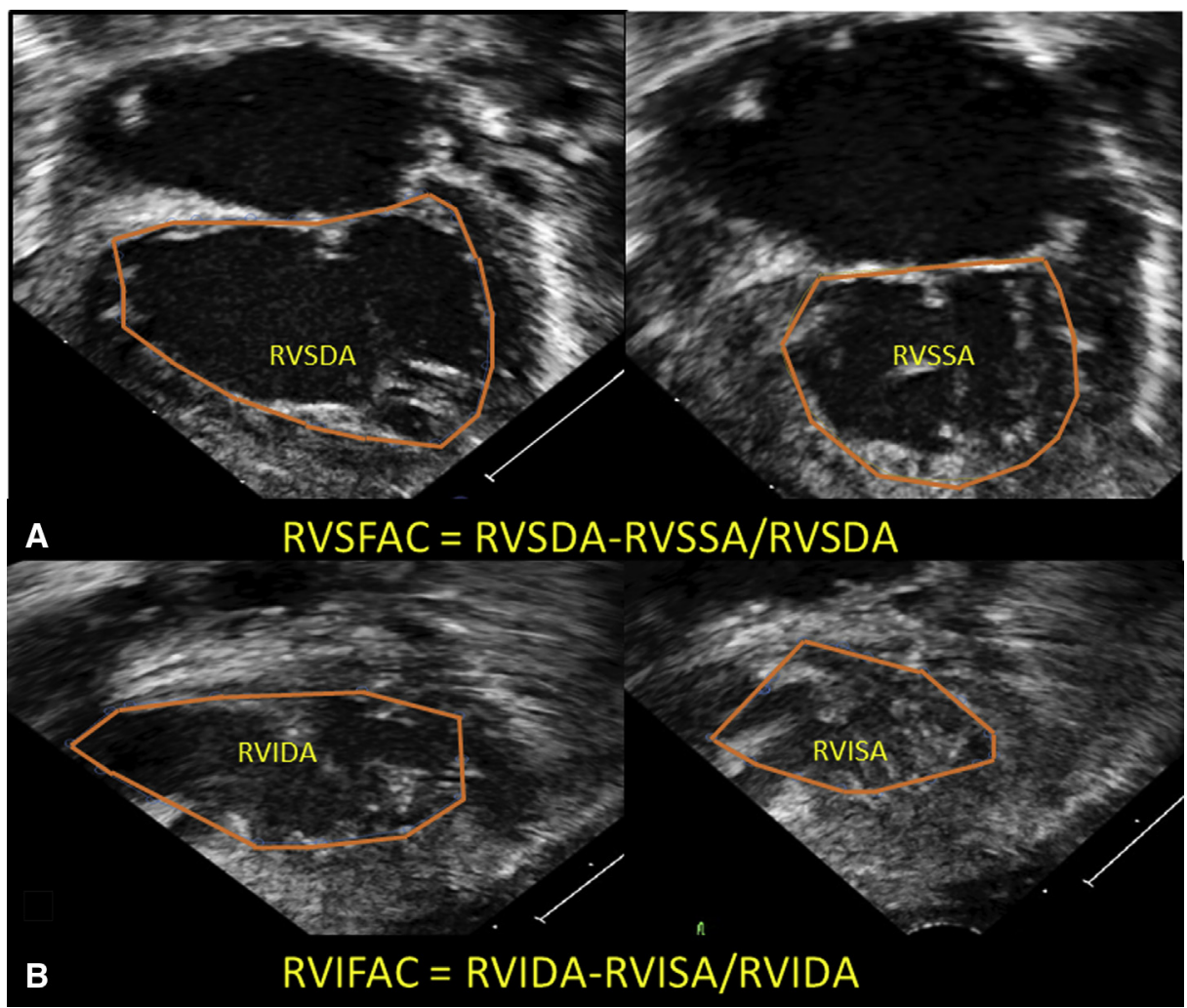

FIGURE 3. Calculation of right ventricle fractional area change $(R V F A C)$. RVFAC was obtained by tracing the RV endocardium on apical images at the RV sinus level (A) as well as the RV infundibulum (B), both at end-diastole (RV sinus diastolic area [RVSDA] and RV infundibular diastolic area [RVIDA]) and end-systole (RV sinus systolic area $[R V S S A]$ and $\mathrm{RV}$ infundibular systolic area $[R V I S A]) . \mathrm{FAC}=100 \times\left(\mathrm{RV}\right.$ diastolic area $\left[\mathrm{cm}^{2}\right]-\mathrm{RV}$ systolic area $\left.\left[\mathrm{cm}^{2}\right]\right) /$ RV diastolic area $\left(\mathrm{cm}^{2}\right)$.

required. This may also contribute to better preservation of myocardial function. This maneuver can also be performed with the heart beating when using either a continuous perfusion or circulatory arrest strategy. In our opinion, the dunk technique involves a bigger incision, greater myocardial stretching, more resection of muscle, and perhaps deeper suturing to the muscle compared with the PT. Ventricular systolic function is a critical determinant in the outcome of patients undergoing single-ventricle palliation. However, echocardiographic assessment of RV systolic function can be challenging, partly due to the lack of adequate visualization of the RV free wall but also because of the complex shape, precluding any geometric assumptions. Various direct and indirect methods of assessment for RV function have been described in the literature with varying success. Amongst these, the RVFAC method has been shown to correlate well with magnetic resonance imaging-derived $\mathrm{RV}$ ejection fractions in adults. ${ }^{16}$ Additionally, the RV area measurements used for RVFAC measurements have also been shown to correlate with magnetic resonance imaging-derived RV volumes in a pediatric study involving tetralogy of Fallot patients. ${ }^{17}$ Furthermore, studies have shown that RVFAC, as a marker of RV function, is diminished in children with pulmonary hypertension when compared with control subjects. ${ }^{18}$ A lower RVFAC in the pre-Norwood stage has also been shown to be independently associated with need for heart transplantation. ${ }^{19}$ Hence, this is a robust and feasible technique to quantify RV systolic function. Moreover, the FAC method can be adopted, both at the RV sinus as well as the RV infundibulum levels (regions that can preferentially get affected from the RV-PA-C insertion site), allowing us to separately quantify RV systolic function in these regions.

Myocardial damage from ventriculotomy in RV-PA-C has known deleterious effects. Although the large Single Ventricle Reconstruction trial showed a significantly better RVFAC in the RV-PA-C group when compared with the MBTT shunt group at the post-Norwood stage, this advantage was no longer significant during the prestage 2 palliation period. ${ }^{20}$ Menon and colleagues ${ }^{5}$ compared the histopathology changes of the RV from autopsies or after heart transplants among patients who received either the RV-PA-C or MBTT shunt during the Norwood procedure and correlated this with echocardiographic findings. They found that all patients who received the RV-PA-C demonstrated scar tissue with thinning and fibrosis in the region 
TABLE 1. Patient characteristics and perioperative data of 22 hypoplastic left heart syndrome patients who underwent Norwood surgery, divided into 2 study groups based on the right ventricle to pulmonary artery conduit (RV-PA-C) placement technique: traditional technique (TT) versus periscope technique (PT)

\begin{tabular}{|c|c|c|c|}
\hline Variable & TT $(\mathbf{n}=8)$ & PT $(n=14)$ & $P$ value \\
\hline Age at Norwood (d) & $3.5(2-18)$ & $5.0(3-18)$ & .57 \\
\hline Age at echocardiogram $(\mathrm{d})$ & $31.5(13-134)$ & $26.5(13-261)$ & .65 \\
\hline Weight at Norwood $(\mathrm{kg})$ & $3.40(2.3-3.6)$ & $3.15(1.9-3.6)$ & .3 \\
\hline Weight at echocardiogram $(\mathrm{kg})$ & $3.70(2.4-7.4)$ & $3.35(2.7-5.4)$ & .19 \\
\hline BSA at echocardiogram $\left(\mathrm{m}^{2}\right)$ & $0.23(0.19-0.28)$ & $0.20(0.16-0.37)$ & $.04 *$ \\
\hline Any RV regional dysfunction grade & $6 / 8(75)$ & $1 / 14(7.1)$ & $.0023^{*}$ \\
\hline I & 2 & 13 & \\
\hline II & 4 & 1 & \\
\hline III & 2 & & \\
\hline RV sinus FAC $(\%)$ & $41(27-49)$ & $50(39-67)$ & $.007 *$ \\
\hline $\mathrm{RV}$ infundibular $\mathrm{FAC} \dagger(\%)$ & $37(7,47)$ & $49(21-64)$ & $.023^{*}$ \\
\hline $\mathrm{CPB}$ duration (min) & $105(90-161)$ & $114(87-145)$ & .4509 \\
\hline Circulatory arrest (min) & $62.5(54-72)$ & $62.5(48-78)$ & .7509 \\
\hline Crossclamp (min) & $62.5(54-72)$ & $63.5(48-78)$ & .9076 \\
\hline HLHS subtype & & & .1684 \\
\hline AA, MS & 2 & & \\
\hline AA, MA & 4 & 11 & \\
\hline AS, MS & 1 & 2 & \\
\hline AA, MS, VSD & 1 & & \\
\hline AS, MA, VSD & & 1 & \\
\hline Pre-Norwood TR & $0 / 8$ & $0 / 14$ & 1.00 \\
\hline None & 1 & 1 & \\
\hline Trivial & 4 & 7 & \\
\hline Mild & 3 & 6 & \\
\hline Pre-Norwood RV dysfunction & $0 / 8$ & $0 / 14$ & 1.00 \\
\hline Neo-aortic valve insufficiency & $0 / 8$ & $0 / 14$ & 1.00 \\
\hline None & 6 & 3 & \\
\hline Trivial & 2 & 8 & \\
\hline Mild & & 3 & \\
\hline Postoperative arrhythmias & $1 / 8(13)$ & $5 / 14(36)$ & .3512 \\
\hline Catheter intervention & $2 / 8(25)$ & $3 / 14(21)$ & 1.00 \\
\hline RV-PA-C/branch PA dilation & 2 & 3 & \\
\hline Mortality & $0 / 8$ & $0 / 14$ & 1.00 \\
\hline
\end{tabular}

Values are presented as median (interquartile range) or $\mathrm{n} / \mathrm{N}(\%) . B S A$, Body surface area; $R V$, right ventricle; $F A C$, fractional area change; $C P B$, cardiopulmonary bypass; $H L H S$, hypoplastic left heart syndrome; $A A$, aortic atresia; $M S$, mitral stenosis; $M A$, mitral atresia; $V S D$, ventricular septal defect; $T R$, tricuspid valve regurgitation. *Statistically significant value. $\nmid \mathrm{RV}$ sinus FAC and RV regional dysfunction pertain to post-Norwood values.

of and surrounding the site of the ventriculotomy and that this correlated with a significantly decreased longitudinal and circumferential myocardial deformation. ${ }^{5}$ Another study looked at heart autopsies of those with HLHS and found increased myocardial fibrosis in hearts that received the RV-PA-C when compared with those who had MBTT shunt and normal hearts. ${ }^{6}$ Furthermore, a study found that on interstage echocardiogram, the RV of those who underwent RV-PA-C when compared with the MBTT shunt had lower ventricular strain and circumferential velocity.
We used echocardiographic data to demonstrate that the limited ventriculotomy and resultant myocardial damage with the PT results in improved RV global and regional systolic function when compared with the TT. Specifically, the qualitative regional dysfunction of the wall segments adjacent to RV-PA-C placement site was only seen in 1 out of 14 patients in the PT group. In contrast, there were 6 out of 8 patients in the TT group that had some degree of regional wall dysfunction $(P=.002)$. RV systolic function quantification also showed a significantly improved median RV 

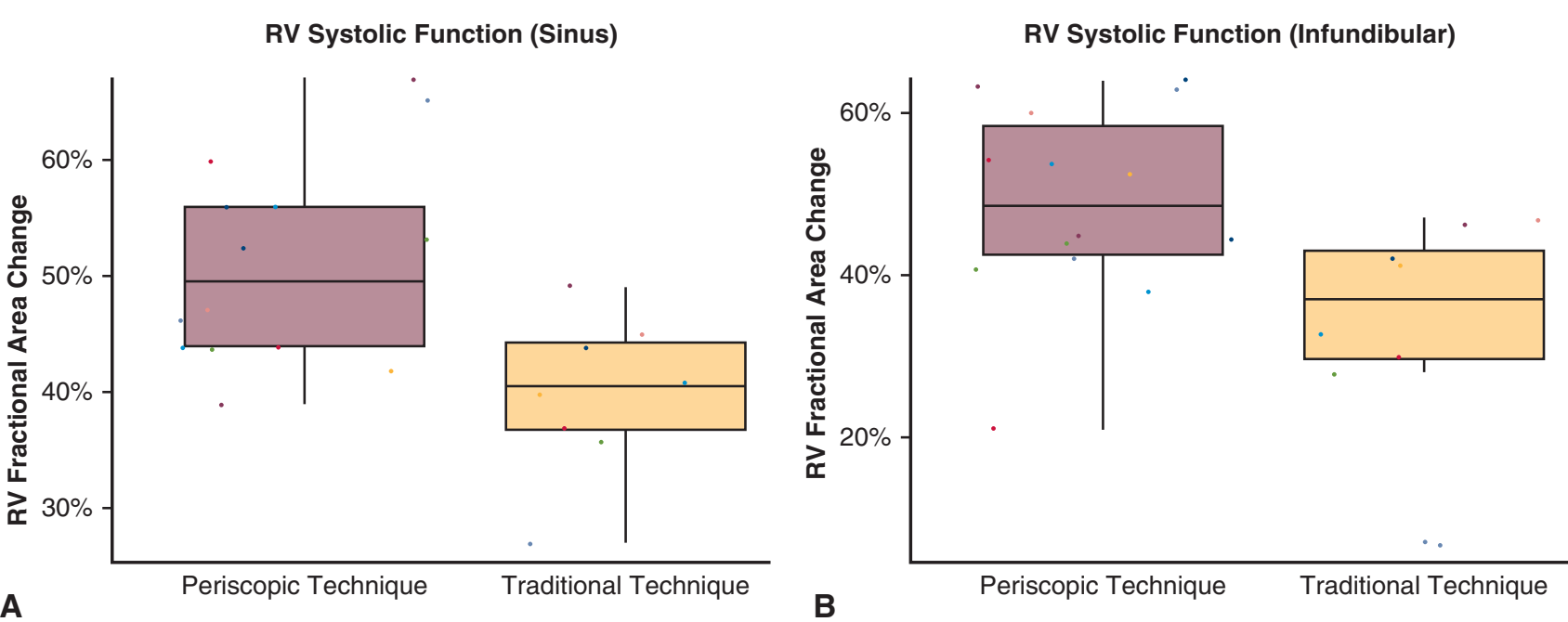

FIGURE 4. Box-plot graph comprising right ventricle $(R V)$ fractional area change values, comparison between periscopic versus traditional technique of right ventricle to pulmonary artery conduit (RVPA-C) placement, both at the RV sinus (A) and the infundibular levels (B). Upper and lower borders of the box represent the upper and lower quartiles. The middle horizontal line represents the median. Each data point is represented by a different color of dot.

infundibular FAC in the PT group (49\%), when compared with the TT group (37\%). This significantly better median RVFAC was also seen at the sinus level: $50 \%$ in the PT group compared with $41 \%$ in the TT group. We hope that our results allow surgeons to consider using the PT for RV-PA-C placement. This may help patients avoid complications of RV dysfunction such as pseudoaneurysm formation, heart failure requiring increased inotropic support, use of extracorporeal membrane oxygenation or ventricular assist device, heart transplants, and death. ${ }^{21-24}$ Although we routinely perform left-sided RV-PA-C, the PT could potentially also be used for right-sided RV-PA-C placement.

\section{Limitations}

Our study is limited by its relatively small sample size $(\mathrm{N}=22)$, although statistically significant results were still seen. We did not assess and correct for any existing baseline (pre-Norwood) differences in the RVFAC amongst the 2 groups, although patients with any baseline RV dysfunction were excluded. The study also included some patients who were younger than age 1 month at the time of the echocardiogram used for RV function assessment, hence not completely correcting for the maturational changes in RVFAC seen during this time period. ${ }^{25}$ Although the echocardiogram reader was blinded to the clinical data, including the type of surgical technique, it is difficult to eliminate the inherent differences in the appearance of the proximal RV-PA-C between the 2 techniques. Additionally, our study examines the early interstage RV function (between stage 1 and stage 2) but does not look beyond this point to provide insights as to how the PT may affect longer-term RV function and clinical outcomes.

\section{CONCLUSIONS}

The novel PT of RV-PA-C placement as part of stage 1 palliation for HLHS is associated with better preservation of early RV global and regional systolic function in our experience, when compared with TT. This preservation of function was seen at both the RV sinus and infundibular regions and likely a result of the technical advantages of a limited ventriculotomy and elimination of myectomy. Whether or not this advantage translates to better longterm preservation in RV systolic function and clinical outcome still needs to be studied.

\section{Conflict of Interest Statement}

The authors reported no conflicts of interest.

The Journal policy requires editors and reviewers to disclose conflicts of interest and to decline handling or reviewing manuscripts for which they may have a conflict of interest. The editors and reviewers of this article have no conflicts of interest.

\section{References}

1. Ohye RG, Sleeper LA, Mahony L, Newburger JW, Pearson GD, Lu M, et al. Comparison of shunt types in the Norwood procedure for single-ventricle lesions. N Engl J Med. 2010;362:1980-92.

2. Pizarro C, Malec E, Maher KO, Januszewska K, Gidding SS, Murdison KA, et al. Right ventricle to pulmonary artery conduit improves outcome after stage I Norwood for hypoplastic left heart syndrome. Circulation. 2003;108(Suppl 1): II155-60.

3. Loomba RS, Shah PH, Chandrasekar S. Short-term outcome comparison of Norwood procedures with right ventricle to pulmonary artery conduit versus modified Blalock-Taussig shunt: a meta-analysis. Ann Pediatr Cardiol. 2011;4:145-9.

4. Sano S, Ishino K, Kawada M, Arai S, Kasahara S, Asai T, et al. Right ventriclepulmonary artery shunt in first-stage palliation of hypoplastic left heart syndrome. J Thorac Cardiovasc Surg. 2003;126:504-9. 
5. Menon SC, Erickson LK, McFadden M, Miller DV. Effect of ventriculotomy on right-ventricular remodeling in hypoplastic left heart syndrome: a histopathological and echocardiography correlation study. Pediatr Cardiol. 2013;34:354-63.

6. Padalino MA, Castellani C, Toffoli S, Della Barbera M, Milanesi O, Thiene G, et al. Pathological changes and myocardial remodelling related to the mode of shunting following surgical palliation for hypoplastic left heart syndrome. Cardiol Young. 2008;18:415-22.

7. Menon SC, Minich LL, Casper TC, Puchalski MD, Hawkins JA, Tani LY. Regional myocardial dysfunction following Norwood with right ventricle to pulmonary artery conduit in patients with hypoplastic left heart syndrome. J Am Soc Echocardiogr. 2011;24:826-33.

8. Lim DS, Peeler BB, Matherne GP, Kramer CM. Cardiovascular magnetic resonance of pulmonary artery growth and ventricular function after Norwood procedure with Sano modification. J Cardiovasc Magn Reson. 2008;10:34.

9. Tweddell J, Mitchell ME, Woods RK, Spray TL, Quintessenza JA. Construction of the RV-PA conduit in the Norwood: the dunk technique. Op Tech Thorac Card Surg. 2012;17:81-98.

10. Tsukashita M, Mosca RS. Periscope modification of right ventricle-to-pulmonary artery shunt in Norwood operation. Ann Thorac Surg. 2014;98:2244-6.

11. Mascio CE, Spray TL. Distal dunk for right ventricle to pulmonary artery shunt in stage 1 palliation. Ann Thorac Surg. 2015;100:2381-2.

12. Newburger JW, Sleeper LA, Frommelt PC, Pearson GD, Mahle WT, Chen S, et al. Transplantation-free survival and interventions at 3 years in the single ventricle reconstruction trial. Circulation. 2014;129:2013-20.

13. Tabbutt S, Dominguez TE, Ravishankar C, Marino BS, Gruber PJ, Wernovsky G, et al. Outcomes after the stage I reconstruction comparing the right ventricular to pulmonary artery conduit with the modified Blalock Taussig shunt. Ann Thorac Surg. 2005;80:1582-90.

14. Kumar TK, Briceno-Medina M, Sathanandam S, Joshi VM, Knott-Craig CJ. Femoral vein homograft as right ventricle to pulmonary artery conduit in stage 1 Norwood operation. Ann Thorac Surg. 2017;103:1969-74.

15. Bentham JR, Baird CW, Porras DP, Rathod RH, Marshall AC. A reinforced rightventricle-to-pulmonary-artery conduit for the stage-1 Norwood procedure improves pulmonary artery growth. J Thorac Cardiovasc Surg. 2015;149: 1502-8.e1.

16. Anavekar NS, Skali H, Bourgoun M, Ghali JK, Kober L, Maggioni AP, et al. Usefulness of right ventricular fractional area change to predict death, heart failure, and stroke following myocardial infarction (from the VALIANT ECHO study). Am J Cardiol. 2008;101:607-12.
17. Alghamdi MH, Mertens L, Lee W, Yoo SJ, Grosse-Wortmann L. Longitudinal right ventricular function is a better predictor of right ventricular contribution to exercise performance than global or outflow tract ejection fraction in tetralogy of Fallot: a combined echocardiography and magnetic resonance study. Eur Heart J Cardiovasc Imaging. 2013;14:235-9.

18. Alkon J, Humpl T, Manlhiot C, McCrindle BW, Reyes JT, Friedberg MK. Usefulness of the right ventricular systolic to diastolic duration ratio to predict functional capacity and survival in children with pulmonary arterial hypertension. Am J Cardiol. 2010;106:430-6.

19. Tweddell JS, Sleeper LA, Ohye RG, Williams IA, Mahony L, Pizarro C, et al Intermediate-term mortality and cardiac transplantation in infants with singleventricle lesions: risk factors and their interaction with shunt type. J Thorac Cardiovasc Surg. 2012;144:152-9.

20. Frommelt PC, Guey LT, Minich LL. Does initial shunt Type for the Norwood procedure affect echocardiographic measures of cardiac size and function during infancy? The single ventricle reconstruction trial. Circulation. 2012; 125:2630-8.

21. Hörer J, Malcic I, Schreiber C, Lange R. False aneurysm origination from the proximal anastomosis of a right ventricular to pulmonary artery shunt following staged repair of hypoplastic left heart syndrome. Interact Cardiovasc Thorac Surg. 2011;12:487-9.

22. Januszewska K, Kozlik-Feldmann R, Dalla-Pozza R, Greil S, Abicht J, Netz H et al. Right ventricle-to-pulmonary artery shunt related complications after Norwood procedure. Eur J Cardiothorac Surg. 2011;40:584-90.

23. Montgomery J, Henderson M, Ostrowsky J, Karimi M, Hennein H. Repair of ventricular aneurysm after stage I modified Sano-Norwood procedure. Crit Care Nurse. 2010;30:54-9.

24. Jean-St-Michel E, Chetan D, Schwartz SM. Outcomes in patients with persisten ventricular dysfunction after stage I palliation for hypoplastic left heart syndrome. Pediatr Cardiol. 2016;37:239-47.

25. Levy PT, Dioneda B, Holland MR, Sekarski TJ, Lee CK, Mathur A, et al. Righ ventricular function in preterm and term neonates: reference values for right ventricle areas and fractional area of change. Am Soc Echocardiogr. 2015;28: 559-69.

Key Words: Norwood, RV function, RV to PA conduit, periscopic technique, echocardiography 